Draft version August 27, 2018

Preprint typeset using $\mathrm{IATEX}_{\mathrm{E}}$ style AASTeX6 v. 1.0

\title{
HABITABILITY IN THE OMEGA CENTAURI CLUSTER
}

\author{
Stephen R. Kane ${ }^{1}$, Sarah J. Deveny ${ }^{2}$ \\ ${ }^{1}$ Department of Earth Sciences, University of California, Riverside, CA 92521, USA \\ ${ }^{2}$ Department of Physics \& Astronomy, San Francisco State University, 1600 Holloway Avenue, San Francisco, CA 94132, USA
}

\begin{abstract}
The search for exoplanets has encompassed a broad range of stellar environments, from single stars in the solar neighborhood to multiple stars and various open clusters. The stellar environment has a profound effect on planet formation and stability evolution and is thus a key component of exoplanetary studies. Dense stellar environments, such as those found in globular clusters, provide particularly strong constraints on sustainability of habitable planetary conditions. Here, we use Hubble Space Telescope observations of the core of the Omega Centauri cluster to derive fundamental parameters for the core stars. These parameters are used to calculate the extent of the Habitable Zone of the observed stars. We describe the distribution of Habitable Zones in the cluster and compare them with the stellar density and expected stellar encounter rate and cluster dynamics. We thus determine the effect of the stellar environment within the Omega Centauri core on the habitability of planets that reside within the cluster. Our results show that the distribution of Habitable Zone outer boundaries generally lie within $0.5 \mathrm{AU}$ of the host stars, but that this small cross-sectional area is counterbalanced by a relatively high rate of stellar close encounters that would disrupt planetary orbits within the Habitable Zone of typical Omega Centauri stars.
\end{abstract}

Keywords: astrobiology - planetary systems - stars: kinematics and dynamics - globular clusters: individual (Omega Centauri)

\section{INTRODUCTION}

Thus far, searches for exoplanets have primarily occurred around field stars, such as the exoplanet survey undertaken by the Kepler mission (Borucki et al. 2010). The prospect of exoplanet detection in globular cluster environments is particularly enticing since they represent a relatively old stellar population and allow studies of how cluster dynamics influences planet formation and evolution (Fregeau et al. 2006; Soker \& Hershenhorn 2007; Spurzem et al. 2009; de Juan Ovalar et al. 2012; Portegies \& Jílková 2015; Cai et al. 2017). A survey for transiting exoplanets among lower main-sequence (MS) stars in the globular cluster NGC 6397 by Nascimbeni et al. (2012) did not detect any significant exoplanet signatures. The primary target of exoplanet searches in globular clusters has been 47 Tucanae (47 Tuc). Observations of 34,000 stars in the 47 Tuc core by Gilliland et al. (2000) using the Hubble Space Telescope (HST) did not detect any transiting planets, despite predictions of almost 20 planet detections. Followup grand-based observations by Weldrake et al. (2005) in the uncrowded outer regions of 47 Tuc also did not de-

skane@ucr.edu tect transiting planets, indicating that the apparent lack of planets in the core may not be solely due to cluster dynamics. However, a recalculation by Masuda \& Winn (2017) of the expected planet occurrence rates in 47 Tuc based on Kepler results determined that only a handful of planets detections should be expected, thus potentially reducing the statistical significance of the initial null result.

Omega Centauri ( $\omega$ Cen, NGC 5139) is a globular cluster that is also the possible remnant of a disrupted dwarf galaxy (Gnedin et al. 2002; Noyola et al. 2008). As the largest globular cluster in the Milky Way galaxy, $\omega$ Cen provides an ideal stellar population for investigations concerning the interaction of radiation environments and stellar dynamics (Merritt et al. 1997; Reijns et al. 2006; van de Ven et al. 2006). An additional advantage of studying cluster stars in the context of exoplanets is that they tend to have measured luminosities that enable the calculation of the Habitable Zone (HZ) for each of the stars (Kopparapu et al. 2013, 2014). Such calculations in turn allow for the quantification of habitability within these dense cluster environments and thus direct the motivation of terrestrial exoplanet searches in globular clusters.

Here we present an analysis of $H S T$ observations of the 
core of $\omega$ Cen and a calculation of HZs for the observed stars. In Section 2 we outline the HST observations, including the calibration, passbands, and quantity of stars. Section 3 describes the methodology used to select MS stars and the derivation of stellar parameters. Section 4 presents the calculations of the HZ for the stellar sample and discusses their distribution. The convolution of the HZ boundaries and the stellar dynamics is addressed in Section 5, taking into account the mean distance between stars and the rate of close stellar encounters. Section 6 discusses the implications of the $\mathrm{HZ}$ calculations for potential habitability within $\omega$ Cen and how this is balanced by planetary orbit disruptions from the close encounter rate between stars. We finally provide concluding remarks in Section 7.

\section{HST OBSERVATIONS OF $\omega$ CENTAURI}

The $\omega$ Cen cluster has been extensively observed by $H S T$ with a variety of science goals using, for example, the Advanced Camera for Surveys Wide Field Channel (ACS/WFC) (Cool 2002). Many groups have performed a wide range of studies that include its multiple stellar populations (Milone et al. 2017), proper motion (Bellini et al. 2018), optical counterparts to X-ray sources (Cool et al. 2013) and the search for the possible intermediate mass black hole (Noyola et al. 2008; Anderson \& van der Marel 2010; Haggard et al. 2013).

The data used for this project was taken from the recently published photometric catalog from Bellini et al. (2017a) which is now the newest and most extensive photometric analysis of $\omega$ Cen ever undertaken. The HST data includes 26 Wide Field Camera 3 (WFC3/UVIS) filters (18 WFC3/UVIS and 11 WFC3/IR), of which we selected two wide-band filters, F438W and F555W, with $34 \times 350$ s and $27 \times 40$ s exposures, respectively. We chose the specific F438W and F555W filters because they are the most comparable to the standard Johnson $B$ and $V$ filters, respectively. They cover roughly a $5^{\prime} \times 5^{\prime}$ field-ofview (FOV) that contains the $2.37^{\prime}$ radius core of $\omega$ Cen with over 470,000 stars.

\section{DERIVATION OF STELLAR PARAMETERS}

The initial stellar sample consisted of 470,000 stars from which we aimed to extract a sample consisting of MS stars in the core. Due to the nature of photometric uncertainties in the crowded core of $\omega$ Cen, we implemented a cut on the stellar sample that was a simple reduction of the null rms error values for the calibrated magnitudes that reduced the list by approximately 12,000 stars. The second step in revising the stellar sample was a cut to only include the core of $\omega$ Cen because we wanted to use a region of relatively uniform stellar density. This was a significant reduction that left us with a total of just over 410,000 stars.

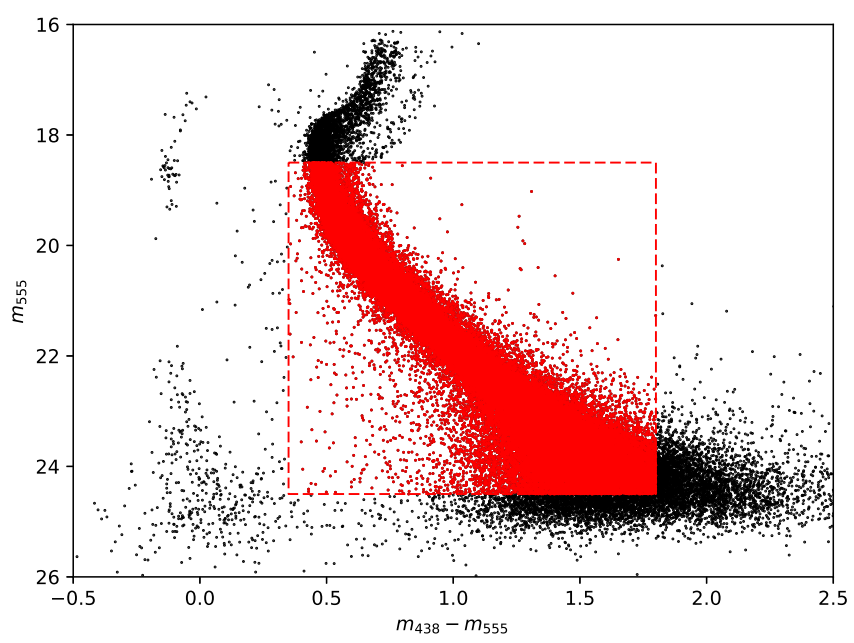

Figure 1. The CMD of the $\omega$ Cen core, including HST photometry of $\sim 410,000$ stars. The red stars inside the dashed box are those selected as MS stars and results in a sample size of $\sim 350,000$ stars.

Finally, we wanted to isolate the MS because habitable zones around giant branch stars or stellar remnants were not what we wanted. This cut was done by using a color-magnitude diagram (CMD) to plot the MS, where the passbands are the F438W and F555W filters described in Section 2. We then manually created boundaries around the MS and selected only the stars that are inside the boundaries. The resulting CMD and MS selection boundaries are shown in Figure 1. The specific cuts for the MS selection are $0.3 \leq m_{438}-m_{555} \leq 1.8$ and $18.5 \leq m_{555} \leq 24.5$. Note that many of the stars at the extreme tail-end of the MS are excluded from our selection due to large error values on the measured F438W and/or F555W magnitudes in that region. After all three of the above cuts were applied, we were left with a total sample size of $\sim 350,000$ stars.

For the subsequent analysis, we required the luminosity $\left(L_{\star}\right)$ and effective temperatures $\left(T_{\text {eff }}\right)$ of the $\omega$ Cen stars. To calculate these stellar parameters, we used an isochrone model of $\omega$ Cen based on the WFC3/UVIS filter system from the "Dartmouth Stellar Evolution Database" (Dotter et al. 2008). The $\omega$ Cen core MS stars have been demonstrated to exhibit a large range of metallicities. Bellini et al. (2017b) described three populations of stars consisting of bMS $([\mathrm{Fe} / \mathrm{H}] \sim-1.4)$, rMS $([\mathrm{Fe} / \mathrm{H}] \sim-1.7)$, and MSe $([\mathrm{Fe} / \mathrm{H}] \sim-0.7)$ stars, where the bMS and rMS stars comprise $\sim 65 \%$ of the core population. We tested the effects of this metallicity diversity on our subsequent $\mathrm{HZ}$ analysis and found that metallicities within the range of rMS to MSe stars have a negligible effect on the HZ calculations. We therefore selected a metallicity of $[\mathrm{Fe} / \mathrm{H}]=-1.49$ (Villanova et al. 2014) since that lies within the metallicity distribution for the bulk of the $\omega$ Cen core stars. We used the 11.5 Gyr age isochrone model, consistent with the age of $11.52 \mathrm{Gyr}$ 

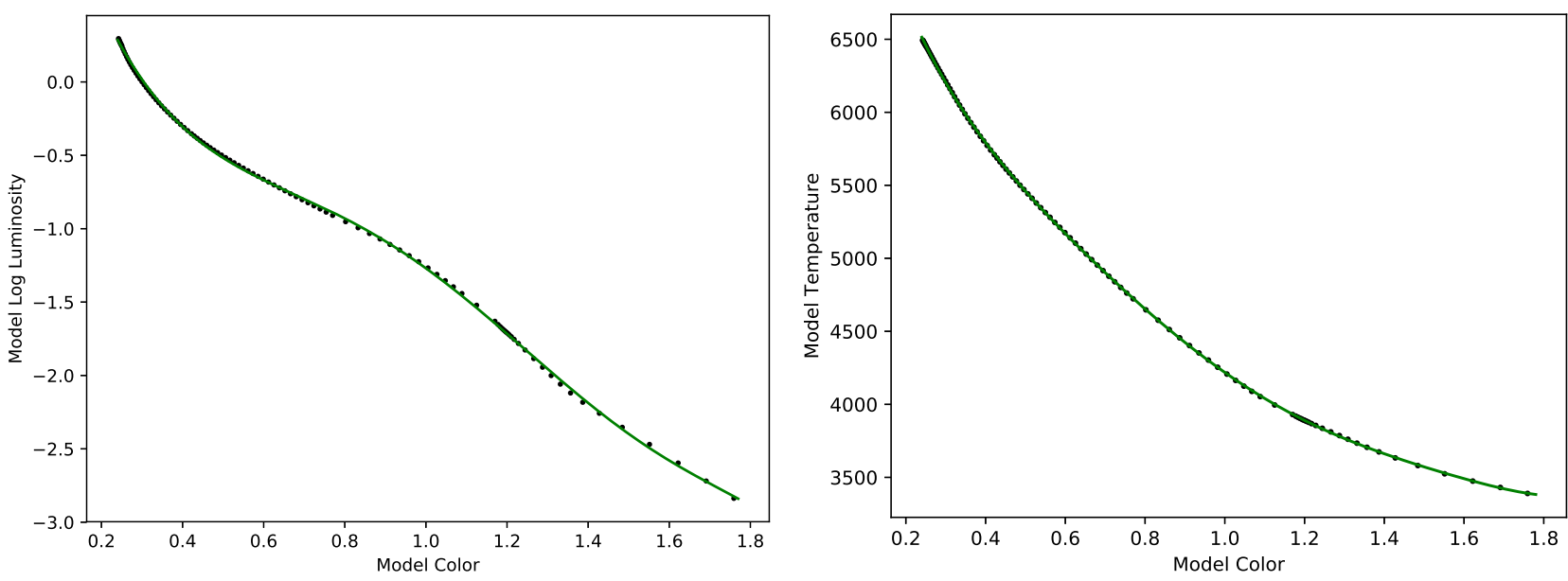

Figure 2. The isochrone model luminosity (left) and effective temperature (right) as a function of the $B-V$ model color. The green solid line shows the result of a sixth-order polynomial fit to the isochrone data, enabling the measurement of luminosity and effective temperature values for the $\omega$ Cen stars observed with HST.

for the age of $\omega$ Cen (Forbes \& Bridges 2010). We then performed a least-squares sixth-order polynomial fit between the $B-V$ colors and the $L_{\star}$ and $T_{\text {eff }}$ isochrone model values. The isochrone data and their associated fits (green lines) are shown in Figure 2, where the left panel shows $L_{\star}$ vs $B-V$ color, and the right panel shows

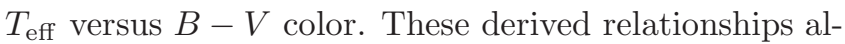
lowed us to calculate the $L_{\star}$ and $T_{\text {eff }}$ values, along with propagated uncertainties, from the colors measured from the $\omega$ Cen HST observations (see Figure 1). We also included the effects of reddening toward $\omega$ Cen in these calculations using the results of the multiband photometry of Calamida et al. (2017). In our sample, $\sim 80 \%$ of the stars have luminosities less than $25 \%$ of solar luminosity, consistent with the relatively large amount of low-mass stars shown in Figure 1 and also with the aged population of the $\omega$ Cen stars.

\section{HABITABLE ZONES IN $\omega$ CENTAURI}

The HZ is generally defined as the region around a star where a terrestrial planet may possibly have surface conditions suitable for liquid water, given sufficient atmospheric pressure. The extent of this region has been quantified by a number of sources, most prominently by Kasting et al. (1993) and further revised by Kopparapu et al. (2013, 2014). The primary boundaries that are used to describe the $\mathrm{HZ}$ are the conservative $\mathrm{HZ}$ (CHZ) that consider theoretical calculations of maintaining temperate surface conditions, and the optimistic HZ (OHZ) that uses empirically derived assumptions regarding the prevalence of surface water on Venus and Mars (Kasting et al. 2014; Kane et al. 2016). The CHZ and OHZ boundaries were used, for example, to create a catalog of Kepler HZ planets by Kane et al. (2016) and are represented graphically for known exoplanetary systems in the Habitable Zone Gallery ${ }^{1}$ (Kane \& Gelino 2012).

Table 1. Measured and derived values for a sample of $\omega$ Cen stars.

\begin{tabular}{ccccccc}
\hline \hline$B-V$ & $L_{\star}\left(L_{\odot}\right)$ & $T_{\text {eff }}(\mathrm{K})$ & $\mathrm{OHZ}_{i}(\mathrm{AU})$ & $\mathrm{CHZ}_{i}(\mathrm{AU})$ & $\mathrm{CHZ}_{o}(\mathrm{AU})$ & $\mathrm{OHZ}(\mathrm{AU})$ \\
\hline $0.438 \pm 0.002$ & $0.413 \pm 0.005$ & $5659 \pm 8$ & $0.486 \pm 0.003$ & $0.615 \pm 0.004$ & $1.089 \pm 0.007$ & $1.149 \pm 0.007$ \\
$0.633 \pm 0.004$ & $0.191 \pm 0.005$ & $5079 \pm 11$ & $0.341 \pm 0.005$ & $0.432 \pm 0.006$ & $0.780 \pm 0.011$ & $0.823 \pm 0.011$ \\
$0.493 \pm 0.022$ & $0.324 \pm 0.045$ & $5481 \pm 69$ & $0.435 \pm 0.030$ & $0.551 \pm 0.038$ & $0.979 \pm 0.068$ & $1.033 \pm 0.071$ \\
$0.905 \pm 0.047$ & $0.081 \pm 0.076$ & $4413 \pm 102$ & $0.228 \pm 0.108$ & $0.288 \pm 0.136$ & $0.535 \pm 0.253$ & $0.565 \pm 0.267$ \\
$0.686 \pm 0.066$ & $0.161 \pm 0.078$ & $4938 \pm 171$ & $0.315 \pm 0.076$ & $0.399 \pm 0.097$ & $0.724 \pm 0.176$ & $0.764 \pm 0.185$ \\
$0.608 \pm 0.056$ & $0.209 \pm 0.077$ & $5147 \pm 153$ & $0.355 \pm 0.065$ & $0.450 \pm 0.083$ & $0.810 \pm 0.149$ & $0.854 \pm 0.158$ \\
\hline
\end{tabular}

Table 1 continued

\footnotetext{
${ }^{1}$ http://hzgallery.org
} 
Table 1 (continued)

\begin{tabular}{ccccccc}
\hline \hline$B-V$ & $L_{\star}\left(L_{\odot}\right)$ & $T_{\mathrm{eff}}(\mathrm{K})$ & $\mathrm{OHZ}_{i}(\mathrm{AU})$ & $\mathrm{CHZ}_{i}(\mathrm{AU})$ & $\mathrm{CHZ}_{o}(\mathrm{AU})$ & $\mathrm{OHZ}_{o}(\mathrm{AU})$ \\
\hline $1.270 \pm 0.087$ & $0.013 \pm 0.211$ & $3801 \pm 102$ & $0.092 \pm 0.759$ & $0.117 \pm 0.961$ & $0.223 \pm 1.840$ & $0.236 \pm 1.941$ \\
$1.180 \pm 0.024$ & $0.022 \pm 0.059$ & $3918 \pm 34$ & $0.120 \pm 0.164$ & $0.152 \pm 0.207$ & $0.289 \pm 0.394$ & $0.305 \pm 0.416$ \\
$0.605 \pm 0.076$ & $0.211 \pm 0.106$ & $5155 \pm 209$ & $0.357 \pm 0.089$ & $0.452 \pm 0.113$ & $0.813 \pm 0.204$ & $0.858 \pm 0.215$ \\
$0.579 \pm 0.042$ & $0.232 \pm 0.062$ & $5228 \pm 116$ & $0.373 \pm 0.050$ & $0.472 \pm 0.063$ & $0.847 \pm 0.114$ & $0.893 \pm 0.120$ \\
$0.699 \pm 0.040$ & $0.154 \pm 0.048$ & $4904 \pm 104$ & $0.309 \pm 0.048$ & $0.392 \pm 0.060$ & $0.712 \pm 0.110$ & $0.751 \pm 0.116$ \\
$0.499 \pm 0.078$ & $0.316 \pm 0.153$ & $5463 \pm 238$ & $0.430 \pm 0.104$ & $0.544 \pm 0.132$ & $0.969 \pm 0.236$ & $1.022 \pm 0.249$ \\
$0.788 \pm 0.082$ & $0.118 \pm 0.103$ & $4682 \pm 199$ & $0.272 \pm 0.119$ & $0.345 \pm 0.151$ & $0.633 \pm 0.277$ & $0.667 \pm 0.292$ \\
$0.502 \pm 0.037$ & $0.312 \pm 0.072$ & $5454 \pm 112$ & $0.427 \pm 0.049$ & $0.541 \pm 0.062$ & $0.963 \pm 0.111$ & $1.016 \pm 0.117$ \\
$0.864 \pm 0.027$ & $0.093 \pm 0.040$ & $4504 \pm 61$ & $0.243 \pm 0.052$ & $0.308 \pm 0.066$ & $0.570 \pm 0.122$ & $0.601 \pm 0.129$ \\
$0.953 \pm 0.022$ & $0.067 \pm 0.041$ & $4312 \pm 46$ & $0.209 \pm 0.063$ & $0.264 \pm 0.080$ & $0.493 \pm 0.150$ & $0.520 \pm 0.158$ \\
$0.904 \pm 0.041$ & $0.081 \pm 0.067$ & $4415 \pm 90$ & $0.228 \pm 0.094$ & $0.289 \pm 0.119$ & $0.536 \pm 0.221$ & $0.565 \pm 0.234$ \\
$0.446 \pm 0.022$ & $0.398 \pm 0.051$ & $5632 \pm 73$ & $0.478 \pm 0.031$ & $0.605 \pm 0.039$ & $1.071 \pm 0.069$ & $1.130 \pm 0.073$ \\
$0.339 \pm 0.008$ & $0.727 \pm 0.022$ & $6030 \pm 33$ & $0.630 \pm 0.010$ & $0.798 \pm 0.012$ & $1.399 \pm 0.021$ & $1.475 \pm 0.023$ \\
$0.711 \pm 0.050$ & $0.149 \pm 0.058$ & $4874 \pm 127$ & $0.304 \pm 0.060$ & $0.385 \pm 0.075$ & $0.700 \pm 0.137$ & $0.739 \pm 0.145$ \\
\hline
\end{tabular}

Using the stellar properties for $\omega$ Cen derived in Section 3, the HZ relationships found in Kopparapu et al. (2014), and the HZ error propagation methodology from Chandler et al. (2016), we calculated the CHZ and OHZ boundaries for each of the stars in our sample. As shown by Kane (2014), the stellar parameter uncertainties can have a significant impact on the determination of $\mathrm{HZ}$ boundaries, thus the need to include the appropriate error propagation in our analysis. A sample of the complete table for our stellar parameters and HZ calculations is shown in Table 1, where the subscripts of $i$ and $o$ are used for the inner and outer HZ boundaries respectively. The distribution of each of the HZ boundaries is represented in Figure 3. The panels of the figure are fixed to identical scales for ease of comparison. The inset panel shows a normalized cumulative histogram of the distribution, where the $\mathrm{x}$-axis is identical to the main plot. The distributions are consistent with the stellar parameters derived in Section 3 which show that the stellar sample is dominated by low-mass stars. For $\sim 50 \%$ of our stellar sample, the outermost HZ boundary $\left(\mathrm{OHZ}_{o}\right)$ lies within $0.5 \mathrm{AU}$ of the star.

\section{MEAN STELLAR DENSITY AND CLUSTER DYNAMICS}

In this section, we calculate an estimate for the mean stellar density of the $\omega$ Cen core. To do this, we adopt the velocity dispersion data for globular clusters provided by Pryor \& Meylan (1993). According to this catalog, $\omega$ Cen has a mean core density of $\sim 3,000 M_{\odot} / \mathrm{pc}^{3}$. Based on the stellar distribution described in Section 3, the mean stellar mass within the cluster is $\sim 0.4 M_{\odot}$. The average volume occupied by a single cluster star is thus $1.3 \times 10^{-4} \mathrm{pc}^{3}$ which results in a mean separation between stars of $0.05 \mathrm{pc}(10,000 \mathrm{AU})$ between stars. These results are consistent with the estimates of the core density by Merritt et al. (1997) using cluster dynamics based on radial velocity measurements.

Numerous observations of $\omega$ Cen, including radial velocities of individual cluster members, have been used to study the dynamics of the core and surrounding regions (Merritt et al. 1997; Reijns et al. 2006; van de Ven et al. 2006). Close encounters between stars can greatly effect the local dynamical environment (Ashurov 2004; Malmberg et al. 2007), particularly the stability of planetary systems (Malmberg et al. 2011) and the potential creation of highly eccentric planetary orbits (Malmberg \& Davies 2009; Kane \& Raymond 2014). Here we utilize the stellar encounter rate methodology discussed by Malmberg et al. (2007) to quantify the potential for close encounters in $\omega$ Cen that may disrupt planetary systems. Specifically, we use Equation 1 of Malmberg et al. (2007) that describes the timescale for a given star to pass within a distance $r_{\text {min }}$ of another star:

$\tau_{\text {enc }} \simeq 3.3 \times 10^{7} \mathrm{yr}\left(\frac{100 \mathrm{pc}^{-3}}{n}\right)\left(\frac{v_{\infty}}{1 \mathrm{~km} \mathrm{~s}^{-1}}\right)\left(\frac{10^{3} \mathrm{AU}}{r_{\min }}\right)\left(\frac{M_{\odot}}{m_{t}}\right)$

where $n$ is the stellar number density, $v_{\infty}$ is the mean relative speed of the stars at infinity, and $m_{t}$ is the total mass of the stars involved in the encounter. The cluster properties described above and the mean stellar mass of 

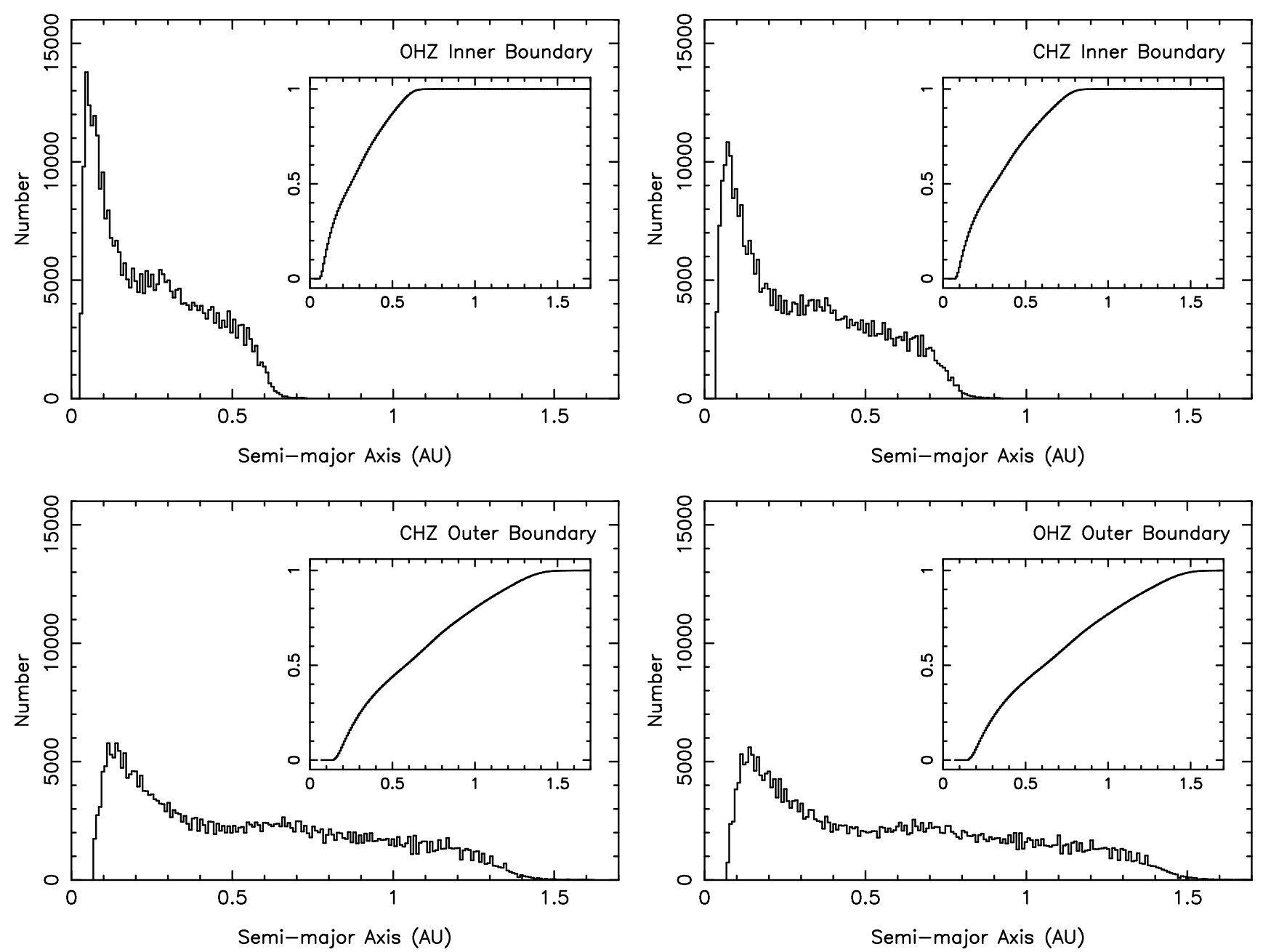

Figure 3. Histograms of the HZ boundaries for the inner OHZ (top-left), inner CHZ (top-right), outer CHZ (bottom-left), and outer OHZ (bottom-right). All four panels use the same axis scales for ease of comparison. The inset panel is a normalized cumulative histogram, where the x-axis is also semi-major axis. The distribution of HZ boundaries matches the distribution of stellar properties derived in Section 3, where the sample is dominated by low-mass stars.

$0.4 M_{\odot}$ result in calculated values of $n=7.5 \times 10^{5}$ and $m_{t}=0.8 M_{\odot}$. Using the velocity dispersion measurements of Pryor \& Meylan (1993), Reijns et al. (2006), and Anderson \& van der Marel (2010), combined with the FOV of the HST observations described in Section 2, we adopt a relative speed for the stars of $v_{\infty}=$ $15 \mathrm{~km} \mathrm{~s}^{-1}$. We then calculate the encounter timescale using Equation 1 as a function of $r_{\min }$.

The results of these calculations are shown as the solid line in Figure 4. The vertical and horizontal dashed lines represent a minimum stellar separation of $r_{\min }=0.5 \mathrm{AU}$, for which the close encounter timescale is $\tau_{\text {enc }}=1.65 \times 10^{6}$ years. The significance of this particular minimum separation is that it corresponds to the distance from the star within which the OHZ outer boundary lies for $\sim 50 \%$ of our stellar sample, as described in Section 4. Notice also that the encounter timescale for $r_{\min }=1,000 \mathrm{AU}$ is only $\sim 1,000$ years, consistent with the mean distance between stars of 10,000 AU. It is worth noting that these encounter timescale calculations include only the effect of the MS stars comprised in our sample, described in Section 3. The inclusion of the red giant branch and white dwarf populations, along with other evolved stars, will have the effect of increasing the total mass of the stars participating in the encounter. According to Equation 1, the result of that inclusion would be to decrease the mean time between close encounters for a given $r_{\min }$.

\section{IMPLICATIONS FOR HABITABILITY}

The search for exoplanets within globular clusters has had a checkered history, such as the interpretation of 47 Tuc observations described in Section 1. As such, the prevalence of exoplanets in these high stellar density environments remains somewhat uncertain. Di Stefano \& Ray (2016) argued that globular clusters 


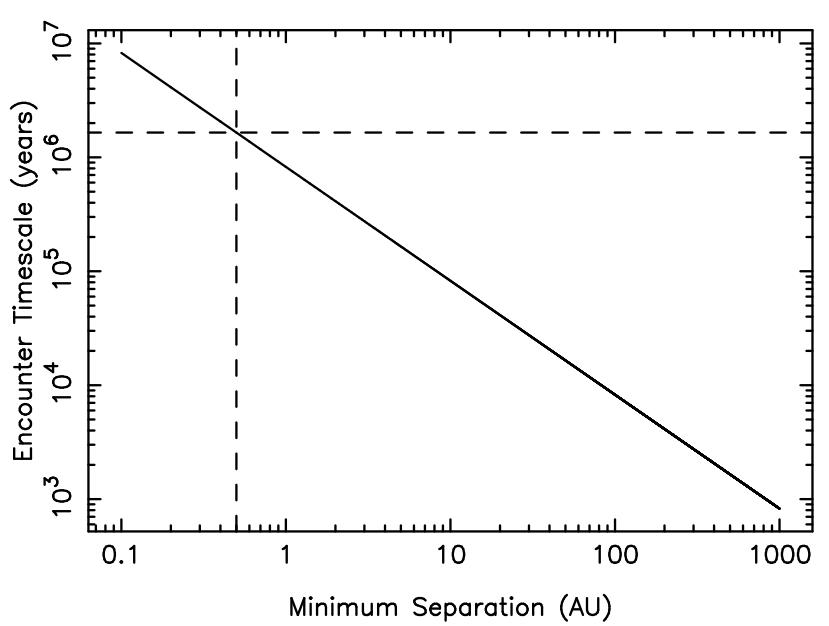

Figure 4. The timescale for a close encounter between two $0.4 M_{\odot}$ stars in the core of $\omega$ Cen as a function of the minimum separation of the stars during the encounter (solid line). The dashed lines indicate the encounter timescale for a minimum separation of $0.5 \mathrm{AU}$, which encompasses the $\mathrm{OHZ}$ outer boundary for $\sim 50 \%$ of the observed stars, as described in Section 4 to locations for expansion of advanced civilizations due to the proximity between stars. The relatively low metallicity of stars in globular clusters could result in a lower occurrence rate of short-period jovian planets (Fischer \& Valenti 2005), although more recent studies of Kepler host star abundances indicate that the occurrence rates of terrestrial planets and Jupiter analogs are less sensitive to host star metallicity (Buchhave et al. 2012, 2018). A study of open clusters observed with Kepler was performed by Chatterjee et al. (2012) and demonstrated that planets detected in these environments could be indicative of perturbations of planetary orbits in globular clusters.

The distribution of HZ boundaries calculated in Section 4 suggests that $\omega$ Cen could be potentially be populated with a plethora of compact planetary systems that harbor $\mathrm{HZ}$ planets close to the host star. An extreme example of such a system is TRAPPIST-1, which contains three planets within the HZ of the host star (Gillon et al. 2017). However, the proximity of the stars combined with the dynamics of the cluster ensure that close encounters between the stars are relatively frequent. As shown in Section 5 and Figure 4, a close encounter of $\sim 1$ AU between typical core cluster members will occur every $\sim 10^{6}$ years on average. Even for a minimum encounter separation of $r_{\min }=0.01 \mathrm{AU}$, comparable to the semi-major axis of the inner planets in the TRAPPIST-1 system, the timescale for such an event is $\sim 10^{9}$ years. The result of these frequent disruptive stellar encounters will be to strip planets from their host stars and create a large population of free-floating terrestrial planets (Malmberg et al. 2011). A large population of free-floating planets has previously been constrained from microlensing observations (Ban et al. 2016; Clanton \& Gaudi 2016) and predicted from core-accretion theory (Ma et al. 2016). Henderson \& Shvartzvald (2016) outlined a strategy through which free-floating planets could be characterized, including planets within the terrestrial regime. Furthermore, Stevenson (1999) proposed that freefloating planets with a rich molecular hydrogen atmosphere can retain habitable conditions at the surface. Thus, despite the dire dynamical environment of the $\omega$ Cen core, habitable planets in that region cannot be entirely ruled out.

\section{CONCLUSIONS}

The $\omega$ Cen cluster is amongst the most studied objects in the sky and provides a unique opportunity to study large globular cluster dynamics as well as the effect on the local group. The HST observations of the core have been utilized here to fully explore the $\mathrm{HZ}$ distribution of the stars in that region and we have presented the first such calculations of HZs in an extremely high stellar density environment. The peak of the HZ distribution within $0.5 \mathrm{AU}$ of the host stars is a consequence of the relatively aged population of stars in the cluster and is a positive aspect of the overall habitability environment in the $\omega$ Cen core. However, the compact nature of the $\mathrm{HZ}$ regions is more than offset by the potential disruption of planetary systems, where close encounters of only $0.5 \mathrm{AU}$ are expected to occur on average every $1.65 \times 10^{6}$ years. Though the large resulting population of free-floating terrestrial planets are intrinsically interesting from formation and dynamical points of view, the potential for habitability in the $\omega$ Cen core environment is significantly reduced by such scattering events. The primary lesson that can be extracted from this analysis is the underlining of the importance of quantifying the long-term dynamical stability of orbits inside $\mathrm{HZ}$ regions taking into account both internal (planetary) dynamics and external (stellar) interactions.

\section{ACKNOWLEDGEMENTS}

The authors would like to thank Adrienne Cool for providing a wealth of valuable feedback, and to the anonymous referee, whose comments greatly improved the quality of the paper. This research has also made use of the Habitable Zone Gallery at hzgallery.org, and the NASA Exoplanet Archive, which is operated by the California Institute of Technology, under contract with the National Aeronautics and Space Administration under the Exoplanet Exploration Program. The results reported herein benefited from collaborations and/or information exchange within NASA's Nexus for Exoplanet System Science (NExSS) research coordination network sponsored by NASA's Science Mission Directorate. 


\section{REFERENCES}

Anderson, J., van der Marel, R.P. 2010, ApJ, 710, 1032

Ashurov, A.E. 2004, AJ, 127, 2154

Ban, M., Kerins, E., Robin, A.C. 2016, A\&A, 595, A53

Bellini, A., Anderson, J., Bedin, L.R., et al. 2017a, ApJ, 842, 6

Bellini, A., Milone, A.P., Anderson, J., et al. 2017b, ApJ, 844, 164

Bellini, A., Libralato, M., Bedin, L.R., et al. 2018, ApJ, 853, 86

Borucki, W.J., Koch, D., Basri, G., et al. 2010, Science, 327, 977

Buchhave, L.A., Latham, D.W., Johansen, A., et al. 2012, Nature, 486, 375

Buchhave, L.A., Bitsch, B., Johansen, A., et al. 2018, ApJ, 856, 37

Cai, M.X., Kouwenhoven, M.B.N., Portegies Zwart, S.F., Spurzem, R. MNRAS, 470, 4337

Calamida, A., Strampelli, G., Rest, A., et al. 2017, AJ, 153, 175

Chandler, C.O., McDonald, I., Kane, S.R. 2016, AJ, 151, 59

Chatterjee, S., Ford, E.B., Geller, A.M., Rasio, F.A. 2012, MNRAS, 427, 1587

Clanton, C., Gaudi, B.S. 2016, ApJ, 834, 46

Cool, A. 2002, "Optical Counterparts for Low-Luminosity X-ray Sources in Omega Centauri", HST Proposal ID 9442. Cycle 11 Cool, A.M., Haggard, D., Arias, T., et al. 2013, ApJ, 763, 126

de Juan Ovelar, M., Kruijssen, J.M.D., Bressert, E., et al. 2012, A\&A, 546, L1

Di Stefano, R., Ray, A. 2016, ApJ, 827, 54

Dotter, A., Chaboyer, B., Jevremović, D., et al. 2008, ApJS, 178, 89

Fischer, D.A., Valenti, J. 2005, ApJ, 622, 1102

Forbes, D.A., Bridges, T. 2010, MNRAS, 404, 1203

Fregeau, J.M., Chatterjee, S., Rasio, F.A. 2006, ApJ, 640, 1086

Gilliland, R.L., Brown, T.M., Guhathakurta, P., et al. 2000, ApJ, 545, L47

Gillon, M., Triaud, A.H.M.J., Demory, B.-O., et al. 2017, Nature, 542, 456

Gnedin, O.Y., Zhao, H., Pringle, J.E., et al. 2002, ApJ, 568, L23

Haggard, D., Cool, A.M., Heinke, C.O., et al. 2013, ApJ, 773, L31

Henderson, C.B., Shvartzvald, Y. 2016, AJ, 152, 96

Kane, S.R., Gelino, D.M. 2012, PASP, 124, 323

Kane, S.R. 2014, ApJ, 782, 111
Kane, S.R., Raymond, S.N. 2014, ApJ, 784, 104

Kane, S.R., Hill, M.L., Kasting, J.F., et al. 2016, ApJ, 830, 1

Kasting, J.F., Whitmire, D.P., Reynolds, R.T. 1993, Icarus, 101, 108

Kasting, J.F., Kopparapu, R., Ramirez, R.M., Harman, C.E. 2014, PNAS, 111, 12641

Kopparapu, R.K., Ramirez, R., Kasting, J.F., et al. 2013, ApJ, 765,131

Kopparapu, R.K., Ramirez, R.M., SchottelKotte, J., et al. 2014, ApJ, 787, L29

Ma, S., Mao, S., Ida, S., Zhu, W., Lin, D.N.C. 2016, MNRAS, 461, L107

Malmberg, D., De Angeli, F., Davies, M.B., et al. 2007, MNRAS, 378,1207

Malmberg, D., Davies, M.B. 2009, MNRAS, 394, L26

Malmberg, D., Davies, M.B., Heggie, D.C. 2011, MNRAS, 411, 859

Masuda, K., Winn, J.N. 2017, AJ, 153, 187

Merritt, D., Meylan, G., Mayor, M. 1997, AJ, 114, 1074

Milone, A.P., Marino, A.F., Bedin, L.R., et al. 2017, MNRAS, 469,800

Nascimbeni, V., Bedin, L.R., Piotto, G., De Marchi, F., Rich, R.M. 2012, A\&A, 541, A144

Noyola, E., Gebhardt, K., Bergmann, M. 2008, ApJ, 676, 1008

Portegies Zwart, S.F., Jílková, L. 2015, MNRAS, 451, 144

Pryor, C., Meylan, G. 1993, ASPC, 50, 357

Reijns, R.A., Seitzer, P., Arnold, R., et al. 2006, A\&A, 445, 503

Soker, N., Hershenhorn, A. 2007, MNRAS, 381, 334

Spurzem, R., Giersz, M., Heggie, D.C., Lin, D.N.C. 2009, ApJ, 697, 458

Stevenson, D.J. 1999, Nature, 400, 32

van de Ven, G., van den Bosch, R.C.E., Verolme, E.K., de Zeeuw, P.T. 2006, A\&A, 513, 513

Villanova, S., Geisler, D., Gratton, R.G., Cassisi, S. 2014, ApJ, 791, 107

Weldrake, D.T.F., Sackett, P.D., Bridges, T.J., Freeman, K.C. 2005, ApJ, 620, 1043 